\title{
Microservices Bad Smells and Automated Detection Tools
}

\author{
Denis Pinto Pinheiro ${ }^{1}$, Eduardo Figueiredo ${ }^{1}$ \\ ${ }^{1}$ Departamento de Ciência da Computação (DCC) \\ Universidade Federal de Minas Gerais (UFMG) \\ Belo Horizonte $-\mathrm{MG}-$ Brasil \\ \{denis.pinheiro, figueiredo\}@dcc.ufmg.br
}

\begin{abstract}
Microservice architectural style is becoming popular in the development of modern applications, but symptoms of good software quality still remain. Code smells and architectural smells (called Bad smells) are identifiable characteristics in software indicating a poor design which might affect the maintainability and understandability. The goal of this master thesis proposal is to present the plan of a review of the literature on the subject of Microservice Bad Smells (MBS) and related MBS detection tools (MBSDT), consolidating the knowledge by compiling a full characterized data set of current identified MBS and evaluate the effectiveness of related existing detection tools.
\end{abstract}

Resumo. Arquitetura de Microserviços é um estilo arquitetural que tem se tornado cada vez mais polupar no desenvolvimento de aplicações modernas, mas sintomas de qualidade software ainda são importantes. Code Smells e Smells Arquiteturais (chamados de Bad Smells) são características identificáveis de projetos de software ruins que podem afetar sua manutenibilidade e compreensão. O objetivo desta proposta de dissertação de mestrado é apresentar um plano de revisão da literatura sobre Bad Smells de Microserviços (MBS) e ferramentas de detecção (MBSDT), consolidando o conhecimento relacionado, compilando um conjunto de MBS identificados e avaliando a efetividade das ferramentas de detecção existentes.

\section{Introduction}

Microservice (MSA) is an architectural approach emerging out of service-oriented architecture, emphasizing self-management and lightweightness as the means to improve software agility, scalability, and autonomy [Jamshidi et al. 2018]. MSA puts a strong emphasis on loose coupling and high cohesion of services, which is considered to result in a better scalability, adaptability, and quality of software architectures [Rademacher et al. 2019]. The MSA style is becoming popular in the development of modern applications, but symptoms of good software quality still remain [Taibi and Lenarduzzi 2018, Bogner et al. 2019].

Code smells and architectural smells (also called bad smells) are symptoms of poor design that can hinder code understandability and decrease maintainability. Several bad smells have been defined in the literature for both generic architectures and specific architectures. However, cloud-native applications based on microservices can be affected by other types of issues [Taibi and Lenarduzzi 2018] and the research field for servicebased antipatterns and bad smells is not as cohesive and organized [Bogner et al. 2019]. 
Given the recent increasing interest on microservices and the quality impact of architecture smells over the microservice-based applications, the main goal of the master thesis is to make a broader review of the literature on the subject of Microservice Bad Smells (MBS) and related MBS detection tools (MBSDT). As a result, it is expected to compile a full characterized data set of current identified MBS and evaluate the effectiveness of related existing detection tools. In the end, the final expected contribution of this master thesis is to have a consolidated knowledge regarding MBS and MBSDT, which might be used as a guideline helping practitioners to identify and avoid bad practices during Microservices development, and also the most used detection tools.

The remainder of this master thesis proposal is as following: Section 2 presents the methodology planned to be followed in order to achieve the intended results. Some preliminary results and related work are highlighted in the Section 3 and Section 4, respectively.

\section{Methodology}

The proposed master project has as main goal to make a broader study of the subject related to Microservices Bad Smells (MBS), which the scientific interest (and software market as well) has increasing in the recent years. In order to achieve this goal, the following methodology is presented (Figure 1):

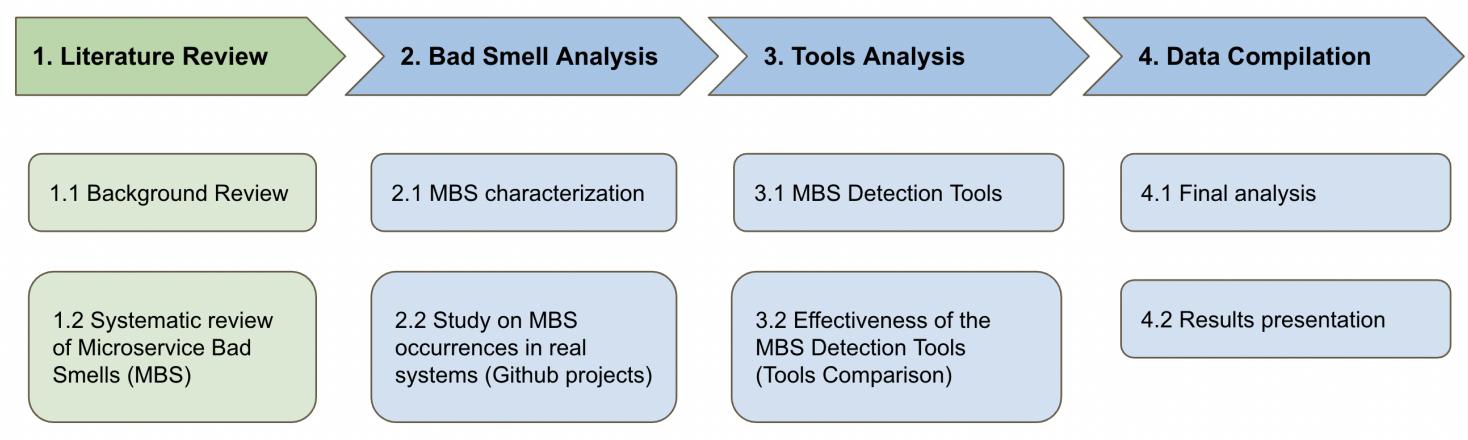

Figura 1. Proposed Master Project Plan

The overall plan has 4 (four) phases:

- Literature Review (on going)

- Microservice Bad Smells Analysis;

- Microservice Bad Smells Detection Tools Analysis; and

- Data Compilation;

\subsection{Literature Review}

The first step is to perform a Literature Review. This phase is an ongoing work and some preliminaries results is presented in the Section 4. The microservice architecture and bad smells theoretical background is being reviewed (step 1.1). Also, a systematic literature review using the Kichenham protocol is being performed in order to identify the MBS and MBSDT in the white literature (step 1.2) [Kitchenham and Charters 2007]. The results of the phase will be used as base for the next steps: (2.) the Microservice Bad Smell Analysis and (3.) the Microservice Bad Smells Detectioin Tool Analysis. 


\subsection{Bad Smells Analysis}

The second phase (2.) goal is to dive deeper into the Microservice Bad Smells (MBS) analysis. Given the MBS set identified in the first phase as a result of the SLR (step 1.2), an overall characterization of the found MBS set (step 2.1) is going to be performed in order to unify taxonomy of different context of microservices smells (eg. code smells, architecture smells, antipatterns, technical debts).

Next (step 2.2), a study of occurrences of the MBS in real systems will be executed. For this end, Github projects implemented using microservice architecture is going to be selected and used to identify MBS instances.

\subsection{Tools Analysis}

The third phase (3.) is planned to analyze the MBS automated detection tools (MBSDT). The first step in this phase (step 3.1), is intended to select the "available" MBSDT identified in the literature. Given the selected MBSDT, the next step is to make an empirical study comparing the tools and evaluating the effectiveness of such tools (step 3.2).

The availability of the MBSDT is a known risk for this phase. If one tool is not open-source and is not available to be downloaded, the authors should be contacted. Another known risk is the stability of the tools: an executable tool with reproducible MBS detection process should be available.

\subsection{Data Compilation}

Finally, the last phase (4.) is to make a compilation of the generated knowledge as result of the MBS and MBST analysis. With this deeper study, the all the scientific knowledge related to MSB and detection tools is going to be compiled and presented. Every phase in this project plan might result in strong scientific contributions.

\section{Preliminary Results}

The ongoing Systematic Literature Review (SLR) using the Kichenham protocol is being performed in order to identify the MBS and MBSDT in the white literature [Kitchenham and Charters 2007]. Following is presented an outline of the steps and preliminaries results achieved.

\begin{tabular}{cc}
\hline Database & Address \\
\hline ACM Digital Library & https://dl.acm.org \\
Engineering Village & https://www.engineeringvillage.com \\
IEEE Explore & https://ieeexplore.ieee.org \\
Science Direct & https://www.sciencedirect.com \\
Scopus & http://scopus.com \\
Springer & http://link.springer.com \\
\hline
\end{tabular}

Figura 2. Digital Libraries

"microservice" AND ( "bad smell" OR "code smell" OR "architectural smell" OR "anti-pattern" OR "technical debt")

Figura 3. Query String 
The research design is comprised of the planning, execution and analysis steps. In the planning the digital libraries (Figure 2) and query string (Figure 3) were defined.

During the execution phase, each step of the search and filtering process is presented in the Figure 4. One of the results is the identification of the interest on the Microservice Bad Smell subject, highlighting when (Figure 5) and where (Figure 6) MBS papers have been published so far.
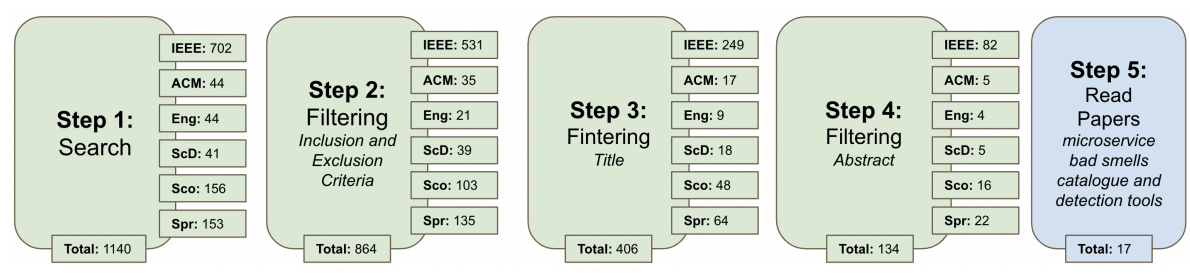

Figura 4. SLR Execution

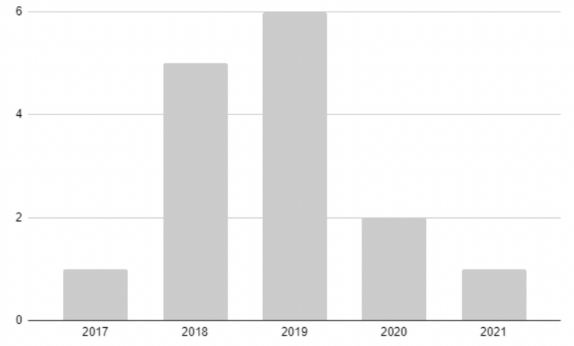

Figura 5. Microservice Bad Smells Interest

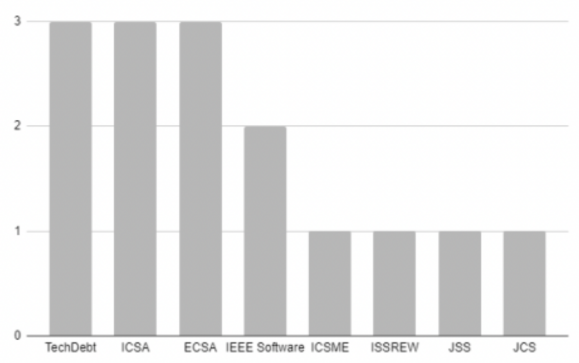

Figura 6. Microservice Bad Smells Publications

\section{Related Work}

Two main works found in the systematic literature review are mainly related to this work and their findings contributed to consolidate a catalog of Microservices Bad Smells and Antipatterns. Taibi and Lenarduzzi (2018) presented a catalog with definitions and guidelines for 11 microservice-specific bad smells as a result of 72 interviews of developers with experience on microservice systems[Taibi and Lenarduzzi 2018].

The second work was Bogner et al. (2019) which performed an SLR focused on service-based anti-patterns, identifying 14 primary studies and reveled in the end 36 anti-patterns and a holistic data model to describe an anti-pattern [Bogner et al. 2019]. 
All secondary works identified during the SLR on Microservices Bad Smells (phase 1 of the project plan - Section 2) have been used to make a comparison of the results achieved so far.

Pereira-Vale et al. (2021) is a recent work focused on investigate security on microservice-based systems, performed a SLR on scientific papers (370) and grey literature (620) in order to provide a comprehensive catalog of security solutions and mechanisms to address security problems on microservice-based systems [Pereira-Vale et al. 2021].

Pigazzini et al. (2020) presented a microservice-specific bad smell detection tool which is capable of identifying tree microservice smells: Cyclic Dependencies, HardCoded and Shared Persistence. The authors affirmed that the work aims to open new perspectives on facing and studying architectural debt in the field of microservices architectures [Pigazzini et al. 2020].

\section{Referências}

Bogner, J., Boceck, T., Popp, M., Tschechlov, D., Wagner, S., and Zimmermann, A. (2019). Towards a collaborative repository for the documentation of service-based antipatterns and bad smells. In 2019 IEEE International Conference on Software Architecture Companion (ICSA-C), pages 95-101.

Jamshidi, P., Pahl, C., Mendonça, N. C., Lewis, J., and Tilkov, S. (2018). Microservices: The journey so far and challenges ahead. IEEE Software, 35(3):24-35.

Kitchenham, B. A. and Charters, S. (2007). Guidelines for performing systematic literature reviews in software engineering. Technical Report EBSE 2007-001, Keele University and Durham University Joint Report.

Pereira-Vale, A., Fernandez, E. B., Monge, R., Astudillo, H., and Márquez, G. (2021). Security in microservice-based systems: A multivocal literature review. Computers Security, 103:102200.

Pigazzini, I., Fontana, F. A., Lenarduzzi, V., and Taibi, D. (2020). Towards microservice smells detection. In Proceedings of the 3rd International Conference on Technical Debt, TechDebt '20, page 92-97, New York, NY, USA. Association for Computing Machinery.

Rademacher, F., Sachweh, S., and Zündorf, A. (2019). Aspect-oriented modeling of technology heterogeneity in microservice architecture. In 2019 IEEE International Conference on Software Architecture (ICSA), pages 21-30.

Taibi, D. and Lenarduzzi, V. (2018). On the definition of microservice bad smells. IEEE Software, 35(3):56-62. 